\title{
A New Based Image Subtraction Algorithm for Bare PCB Defect Detection
}

\author{
Daniel Katz Bonello, Yuzo Iano, Umberto Bonello Neto \\ University of Campinas, Brazil
}

\begin{abstract}
Various concentrated work has been developed in the area of computer vision applied to detection of failures on printed circuit boards ( $P C B$ 's), aiming at reducing the possibility of the occurrence of the fabrication defects. In this research, based on PCI's - without mounting reference and test layout models, the objective is to study is the application of an image subtraction technique to the failure detection of those bare printed circuit boards layouts using image subtraction techniques during the image processing. By developing the primary subtraction algorithm, one may compare the efficacy of this image processing technique using linear simulations developed in MATLAB.
\end{abstract}

\section{Introduction}

According to the [1], printed circuit boards are rigid or semi-rigid boards on which geometrical patterns are printed in copper or some other conductive material. They function is to replace the wiring and perhaps some of the electrical circuit components in everything from toasters to fighter planes. Printing a wire can be less expensive than fitting a real one and soldering it.

The automated inspection of printed circuit boards (PCB's) serves a purpose which is traditional in computer technology. The purpose is to relieve human inspectors of the tedious and inefficient task of looking for those defects in PCBs which could lead to electrical failure. Automated, computer based, inspection relieves this problem by providing a machine solution.

Even, according to IPC (Association Connecting Electronics Industries), the total North American PCB shipments in November 2017 were up $4.0 \%$ compared to the same month in 2016. This year to date, shipments are $2.3 \%$ below the same period last year. Compared to the preceding month, November shipments increased $0.4 \%$ (information data, obtained from http://pcb.iconnect007.com/index.php/article/108110/n orth-american-pcb-industry-growth continues/108113/ ?skin=pcb in January 2018). This not counting the global shipments growth, that motivates the manufacturing investments designed to the PCB's production, also increasing the demand for PCB's inspection processes with reduced inspection time and high-efficacy failures classification to attend the high demand of those products.
Certainly, some inspection technique for reduce the bare PCB's image processing time and enhance the failures classification would be profitable, so decreasing the probabilities of a Company ship a defective product to the final consumers, that would not be economically viable.

In this research article, the main focus is to develop a bare PCB inspection technique through an image subtraction algorithm using a new image subtraction technique viewing the optimization of time reduction and image classifying efficacy of those bare PCB's inspection processes, adopting a theorical and practical approach, as will be explained in the following subtopics of this paper.

\section{Related Works}

Further taking the model utilized by [2], in this work is still utilized a PCB production sample model (contemplating the quantities of interest of this work and of proper bare PCB design layout employed in this analysis), from which is derivate the PCB's digital image layout (binary image) through elementary image processing steps: convert the RGB image to gray scale, thresholding, convert to binary image. Also is described those image processing steps in detail in the next subtopics as well as the main concepts of XOR operation utilized in this work.

\subsection{The RGB Color Model}

According to [3], the coordinates of RGB value is indicated in Figure 1.

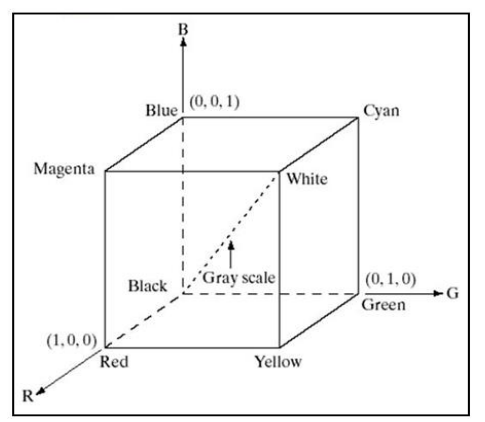

Figure 1. Schematic of the RGB color tube 


\subsection{Gray-scale Images}

Image formation using sensor and other image acquisition equipment denote the brightness or intensity I of the light of an image as two dimensional continuous function $\mathrm{F}(\mathrm{x}, \mathrm{y})$ where $(\mathrm{x}, \mathrm{y})$ denotes the spatial coordinates when only the brightness of light is considered. Sometimes three-dimensional spatial coordinates are used. Image involving only intensity are called gray scale images [4]. A representation of a bare PCB production sample in gray scale is illustrated in Figure 2.

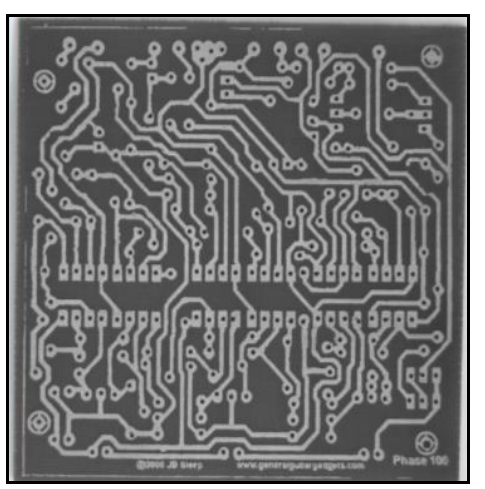

Figure 2. Bare PCB production sample in gray-scale

\subsection{Thresholding Techniques}

According to [3], the gray-level image shown in Figure 2.2 of previous subtopic corresponds to an image, $\mathrm{f}(\mathrm{x}, \mathrm{y})$, composed of light objects on a dark background, in such a way that object and background pixels have gray levels grouped in two dominant modes. One obvious way to extract the objects from the background is to select a threshold $\mathrm{T}$ that separates these modes. Then any point $(x, y)$ for which $f(x, y)>T$ is called object point; otherwise, the point is called background point.

Is initiated the deduction of thresholding equations defining the object of study (bare PCB production sample) as being processed by multiple thresholding techniques, as shows the Figure 3 [5].

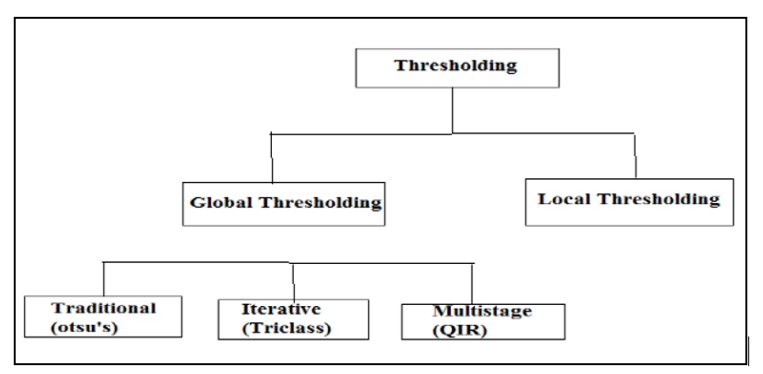

Figure 3. Thresholding techniques

\subsection{Image Binarization}

To simplifying purposes, will be presented here the general concepts of bare PCB's binarization process with an example of a bare production PCB sample binarized from the correspondent thresholded image. Then, taking the bare PCB product sample obtained in the Local Thresholding process, using the Bernsen's Technique, we have the binarizated PCB image in Figure 4.

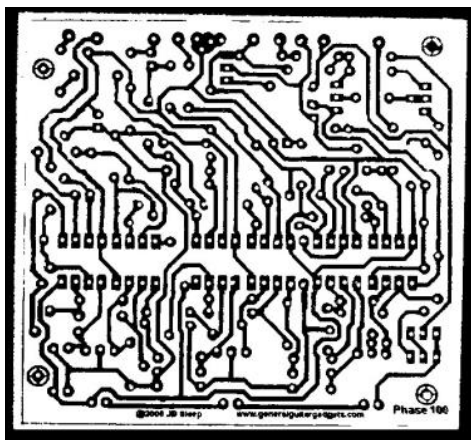

Figure 4. Bare PCB production binarization

\subsection{The XOR Operation In The Image Acquisition Process}

Is initialized now the modeling step of XOR logic operation in the image subtraction process, considering the previous techniques demonstrated in the presented subtopics of this Chapter, such as: RGB color model, Gray-scale image level, Thresholding techniques and the Image binarization method. Therefore, based on reference [3] and [6], the main concepts of XOR operation in the image subtraction process is presented next.

The difference between two images $\mathrm{f}(\mathrm{x}, \mathrm{y})$ and $\mathrm{h}(\mathrm{x}, \mathrm{y})$, can be expressed as the Eq.(1):

$$
G(X, Y)=\mathrm{F}(\mathrm{X}, \mathrm{Y})-\mathrm{H}(\mathrm{X}, \mathrm{Y})
$$

A new image using XOR operation is mostly used for change detection $\mathrm{C}=\mathrm{A}-\mathrm{B}$ : maximum value of $\mathrm{A}-\mathrm{B}$ and zero.

In the XOR operation, if the pixels of Image A and Image $\mathrm{B}$ are complementary to each other than the resultant image pixel is black, otherwise the resultant image pixel is white. The XOR operation of images A and B is showed in Figure 5.

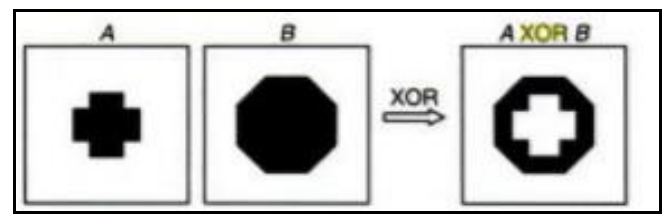

Figure 5. Image XOR operation 
The boolean operations utilized in the image subtraction are represented in Table 1.

Table 1. Boolean logical operation

\begin{tabular}{cccccc}
\hline \multicolumn{3}{c}{ XOR } & \multicolumn{3}{c}{ XNOR } \\
\hline A & B & Q & A & B & Q \\
0 & 0 & 0 & 0 & 0 & 1 \\
0 & 1 & 1 & 0 & 1 & 0 \\
1 & 0 & 1 & 1 & 0 & 0 \\
1 & 1 & 0 & 1 & 1 & 1 \\
\hline
\end{tabular}

\subsection{Previous Studies Comparison}

To effect of previous studies comparison, the results of work [7] will be analyzed, exposing in this way the main advantages/gains of this current developed work.

2.6.1 Results comparison with article "PCB Defect Detection Using Image Subtraction Algorithm". In the Part V (Process Flowchart) of article [7], is exposed the Process B: Converting to gray scale regarding to the transformation two green $\mathrm{PCB}$ production samples as we can see in Figure 6.

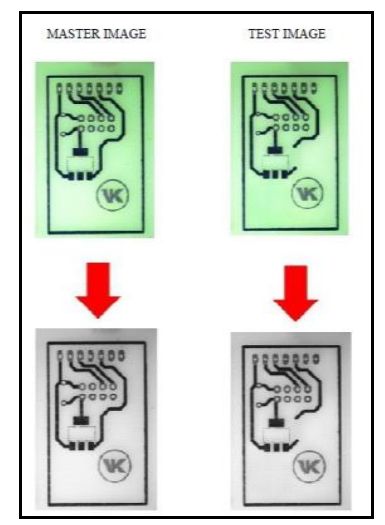

Figure 6. Converting to gray-scale

The grayscale image converting method of the work [7] is based on the increasing contribution of the green color as we can see in the image above. Though it represents an advantage in terms of pixel optimization, and low computer memory storage, it is more convenient utilize a RGB bare PCB image to allows the image subtraction algorithm detect defects from a thresholding value of 128 . Besides, the method of the work [7] utilizes three individual steps before XOR operation: A. Loading The Image, B. Converting to gray scale and $\mathrm{C}$. Thresholding.

In this new based image subtraction algorithm of this manuscript, those three main steps are located in one single step (the pre-algorithm), indicating operational advantages during the bare PCB inspection process. The Figure 7 shows the result obtained for a new GUI image processing utilizing Matlab R2017b as follow bellow:

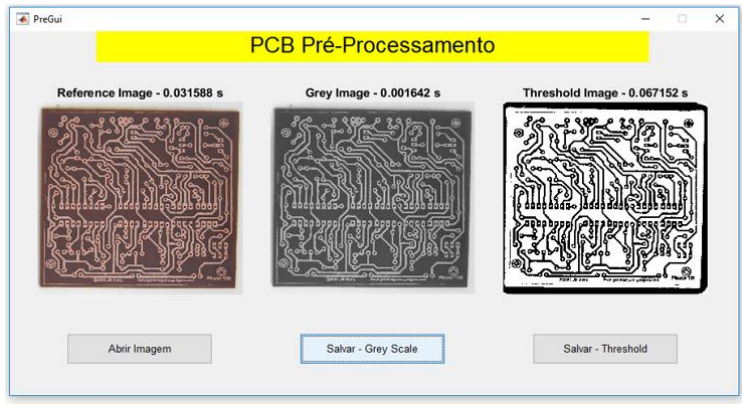

Figure 7. New based GUI image processing

\section{Proposed Method}

\subsection{Resultant algorithm for image pre- processing}

The resultant algorithm obtained from the GUI image processing is represented in the Figure 8 bellow:

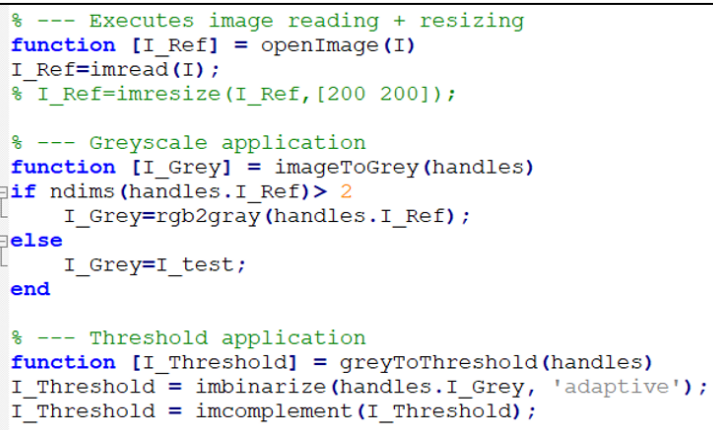

Figure 8. GUI image pre-processing algorithm

\subsection{Resultant algorithm for image subtraction classification}

The resultant algorithm obtained from the PCB image defect class is represented in Figure 9.

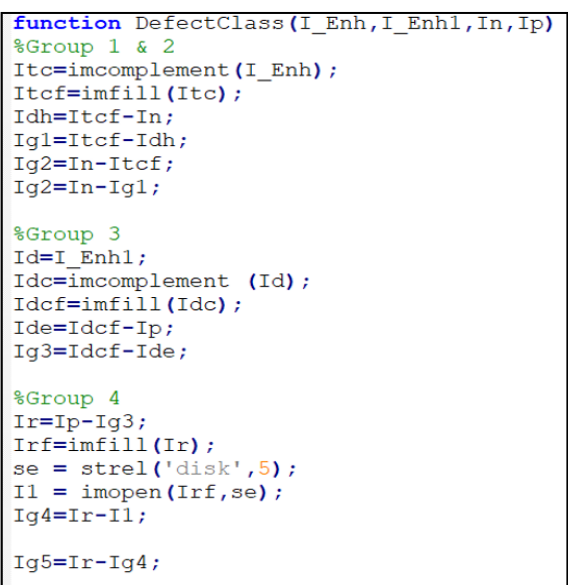

Figure 9. PCB image defect class algorithm 


\subsection{Image processing time and classification efficacy}

3.3.1. Image processing time. According to [8], the inspection time for PCB image binarization it's about 0.078 s utilizing a classical approach in Matlab. In the new based algorithm, the binarization of the reference bare PCB image was about $0.067 \mathrm{~s}$ as we can see in the Figure 7 in the subtopic 2.6.1. It represents a percentage difference about $14.1 \%$ as shows in Grap 1 .

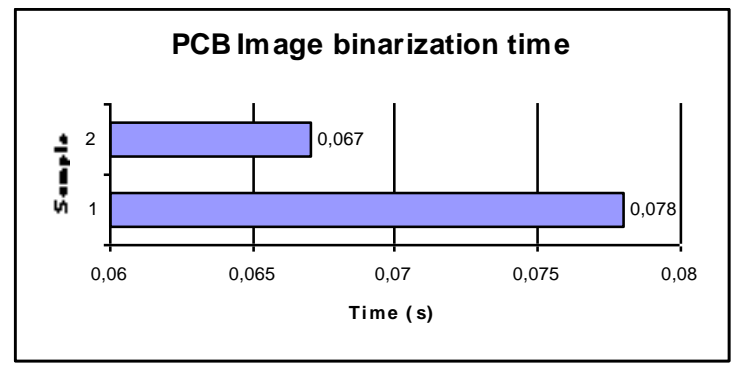

Graph 1. PCB image binarization time

3.3.2. Classification efficacy. The resultant image subtraction algorithm, using XOR operation, can detect four groups of defects and classify him in Table 2.

Table 2. Groups of defects

\begin{tabular}{cc}
\hline ITEM & DEFECT \\
1 & Breakout \\
2 & Pin Hole \\
3 & Open Circuit \\
4 & Underetch
\end{tabular}

The fourth group of defects detected in bare PCB samples are represented in Figure 10.

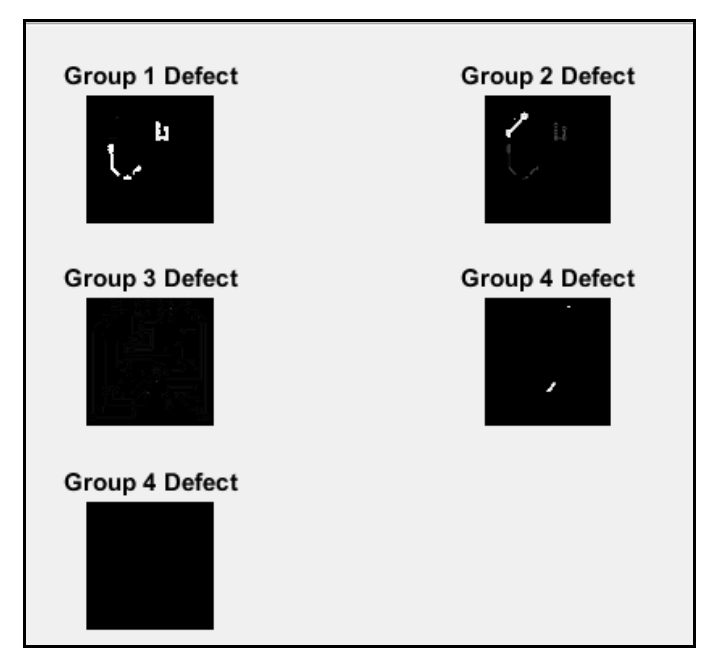

Figure 10. Bare PCB defect classification

\section{Discussion}

The idea of this article is to provide to the reader a general vision about the basics phenomena involved in the image generation process of an image acquisition system. It is important that reader has a basic knowledge about the image acquisition devices, because those are the elements responsible to the PCB's image processing generation. The presentation of elements related to the image acquisition system in this paper allows that your modeling can be incorporated to the wavelets equations of image subtraction technique. The literature regarding to the image acquisition systems is very vast [9].

\section{Conclusion}

In this work was deduced a model commonly utilized in the field of image processing to represent the image subtraction process of a bare PCB production sample. Once defined the pre-processing algorithm and image classification algorithm, were defined optimized implementation strategies of PCB image binarization time with time percentage reduction dependents of the groupment of three preprocessing steps. After a definition of an optimization metric through the new based algorithm for image binarization, was verified the percentage time reduction in the $\mathrm{PCB}$ image binarization, around $14.1 \%$ in comparison of the better result obtained in the work [8]. With this result, was possible obtain also an image classification efficacy in function of the optimized PCB binarized image.

\section{Acknowledgements}

I thank God by Always help me reach my objectives. To my family, by unconditional support demonstrated in all moments during my Master Degree. To the research team of Laboratory of Communications (LCV). To my friends that always be present during my Master Degree. To all professor and employees of School of Electrical and Computer Engineering (FEEC) that has helped me during the development of this work, always ready to give me help. To all the people that contributed in a directly or indirectly way for that this work would be realized.

\section{References}

[1] Thibadeau, R., Printed circuit board inspection. In: Carnegie Mellon University, 1981.

[2] Ortega et al., PCB Inspection Using Image Processing and Wavelet Transform. In: ResearchGate, 2007. 
[3] Gonzalez, R.C., Woods, R.E., Digital Image Processing - 3rd edition. Pretince Hall 2010; 122 pages.

[4] Kumar and Verma, Color-to-grayscale conversion to maintain discriminability. In: Proceedings of SPIE - The International Society for Optical Engineering, 2004.

[5] Senthilkumaran, N., and Vaithegi S., Image Segmentation By Using Thresholding Techniques For Medical Images.

[6] Jayaraman et al., Scilab Textbook Companion for Digital Image Processing. Tata McGraw 2010; 112 pages.

[7] Suhasini, A., et al., PCB Defect Detection Using Image Subtraction Algorithm. In: IJCST, 2015.

[8] Santoyo, et al., PCB Inspection Using Image Processing and Wavelet Transform. In: Mexican International Conference on Artificial Intelligence, 2007.

[9] Tatibana, M.H., Lotufo R., Novel automatic PCB inspection technique based on connectivity. In: Computer Graphics and Image Processing, 1997.

[10] Batchelor, B.G., Whelan, P.F., Intelligent vision systems for industry. Springer 1997; 457 pages. 